\title{
Comprehensive family therapy: an effective approach for cognitive rehabilitation in schizophrenia
}

This article was published in the following Dove Press journal:

Neuropsychiatric Disease and Treatment

21 May 2015

Number of times this article has been viewed

\author{
Jun Cai' \\ Yi Zhu' \\ Weibo Zhang' \\ Yanfeng Wang' \\ Chen Zhang ${ }^{2}$ \\ 'Center for Disease Control \\ and Prevention, Shanghai Mental \\ Health Center, ${ }^{2}$ Schizophrenia \\ Program, Shanghai Mental Health \\ Center, Shanghai Jiao Tong University \\ School of Medicine, Shanghai, People's \\ Republic of China
}

Background: Antipsychotic medication has limited abilities to improve the cognitive impairments that accompany schizophrenia. Adding psychosocial treatment may result in marked improvements in cognitive function, as compared to antipsychotic treatment alone. We hypothesized that a combination of individual and family interventions may be a useful cognitive rehabilitation paradigm for schizophrenia.

Materials and methods: An 18-month follow-up clinical trial of 256 stabilized patients with schizophrenia at six communities in Shanghai, People's Republic of China were randomly assigned to into either a comprehensive family therapy (CFT) group or a usual daily care (UDC) group. The Repeatable Battery for the Assessment of Neuropsychological Status (RBANS) and the Positive and Negative Syndrome Scale (PANSS) were the primary outcome instruments for this study.

Results: There was no significant difference between the CFT and UDC for all demographic characteristics at the baseline assessment. During the 18-month follow-up observation, changes in RBANS total score indicated that patients undergoing CFT showed greater improvement from baseline to the follow-up assessments in cognitive function than those in the UDC group $(F=9.77$, $P=0.002)$. Post hoc analysis showed that the CFT group presented with significant differences in the RBANS total score, immediate memory, visuospatial skill, language, attention, and delayed memory sections compared with the UDC after 18 months of follow-up (all $P<0.01$ ).

Conclusion: Our findings suggest that CFT can be easily adapted and may prove to be an effective approach for improving cognitive function in patients with schizophrenia. Our program provides a potential paradigm for cognitive rehabilitation for schizophrenia patients in the community.

Keywords: cognitive function, comprehensive family therapy, schizophrenia

\section{Introduction}

Schizophrenia is one of the most common and severe forms of mental illness, and is often chronic, recurrent, disabling, and debilitating. ${ }^{1}$ In the People's Republic of China alone, a recent meta-analysis placed the lifetime prevalence risk at 5.44 of every 1,000 people, ${ }^{2}$ making it a significant health care issue in terms of sheer numbers. Moreover, around $80 \%$ of patients with the disease struggle with a variety of neurocognitive deficiencies, including speed of processing, attention/vigilance, working memory, verbal learning, reasoning, and problem solving. ${ }^{3}$ A growing number of studies have indicated that cognitive dysfunction is among the most important factors in contributing to the social deficits and the functional outcome of schizophrenia patients; ${ }^{4}$ moreover, our previous work demonstrated a wide range of cognitive functions that were substantially impaired among antipsychoticnaïve patients with first-episode schizophrenia, ${ }^{5,6}$ which supports the view that cognitive
Correspondence: Chen Zhang Schizophrenia Program, Shanghai Mental Health Center, Shanghai Jiao Tong University School of Medicine, 600 Wan Ping Nan Road, Shanghai 200030, People's Republic of China Tel +8621 64387250 Email zhangchen645@gmail.com 
impairment is at the core of this disorder. ${ }^{7,8}$ Collectively, these different lines of evidence only highlight some of the reasons why finding more effective methods of cognitive improvement has become an increasingly important target for research into schizophrenia and schizophrenia treatment. ${ }^{9}$

From a pharmacological point of view, neither typical nor atypical antipsychotics have shown much promise in their efficacy at combating the cognitive impairment that many schizophrenia patients face. ${ }^{10,11}$ Accordingly, researchers and clinicians have pursued individual interventions aside from medication, including computerized cognitive training, ${ }^{12,13}$ social skills training, ${ }^{14}$ and vocational rehabilitation training, ${ }^{15}$ all of which have been respectively reported to possess certain advantages over more antipsychotic medication. More interestingly, another encouraging approach in recent years has been modifying the living environments of patients with schizophrenia. Friedman-Yakoobian et $\mathrm{al}^{16}$ developed a family-based cognitive adaptation strategy to train family members in how to implement and provide the necessary environmental supports for patients to compensate for their cognitive impairments. These findings showed that family intervention may be feasible treatment options for the cognitive dysfunction that accompanies schizophrenia, and also that both individual and family interventions have the potential to be effective cognitive rehabilitation options.

Here, we hypothesized that a combination of individual and family interventions may be a useful cognitive rehabilitation paradigm for schizophrenia patients in a community setting. In this study, we report on an 18-month follow-up clinical trial that examined the effectiveness of comprehensive family therapy (CFT) on cognitive rehabilitation - including both individual and family interventions - in stabilized patients with schizophrenia.

\section{Materials and methods Subjects}

We carried out this multicenter, parallel-group, randomized controlled trial between January 2013 and December 2013 at six communities in the districts of Baoshan, Changning, Xuhui, Congmin, Jingan, and Songjiang in Shanghai City, People's Republic of China. A total of 256 schizophrenia patients were recruited on the basis of the following criteria: 1) a diagnosis of schizophrenia according to the Diagnostic and Statistical Manual of Mental Disorders, Fourth Edition; ${ }^{17}$ 2) a duration of illness less than 5 years; 3 ) aged between 18 and 60 years; 4) has a minimum education of primary middle school; 5) receives atypical antipsychotic monotherapy; 6) has maintained a stable condition for more than 6 months before entry into the study; 7) has a Positive and Negative Syndrome Scale (PANSS) total score less than $60 ; 8$ ) currently lives with his/her family members, who can help him/her with rehabilitation practice; and 9) has no other observable physical disease or other psychiatric disorder aside from schizophrenia.

All subjects provided written informed consent prior to any procedure related to this study being performed. All procedures of this study were reviewed and approved by the Institutional Review Boards of the Shanghai Mental Health Center, and performed in strict accordance with the Declaration of Helsinki and other relevant national and international regulations.

\section{Study design}

This study was a randomized controlled trial. Using the Statistics Analysis System (SAS) software, the randomization table was generated with a randomization procedure provided by an independent statistician who had no information about the study subjects. ${ }^{18}$ Each subject was randomly allocated to either the CFT group or the usual daily care (UDC) group.

\section{Intervention}

Both the CFT and UDC groups continued their prescribed medications. CFT was carried out on patients as well as their family members, as described here: 1) social skills individualized training (SSIT) was provided to the patients, which is a therapy based on "social and independent living skills", as outlined by Liberman et al. ${ }^{19}$ It includes five modules: medication management; symptom management; community re-entry; recreation for leisure; and social independent living skills. The training was carried out in each community by professional personnel; the methods included watching videos, attending lectures, engaging in group practice and role play, and completing assignments after class. The duration of the training is 90-120 minutes/session, two sessions/ week for 10 weeks. 2) The family intervention to the family members was as follows: psychoeducation was provided by psychiatric health professionals to the patients' family members, including education regarding the causes, symptoms, treatments, diagnoses, and treatment of schizophrenia, as well as the common adverse effects of antipsychotics, and the prognosis and prevention of schizophrenia. The therapy was conducted in one session a week for 10 consecutive weeks.

During the study, patients in the UDC group continued to receive treatment as usual, which generally consisted of a monthly outpatient service. They received antipsychotic medication at a dosage that could be changed when clinically 
required. UDC was delivered before and throughout the study; it was thus hypothesized to be ineffective on outcome measures.

Booklets containing useful information and regular consulting activities were made available to caregiving family members. The presence of patients and their family members was recorded, and additional training was given in the event of absences.

\section{Evaluation}

The Repeatable Battery for the Assessment of Neuropsychological Status (RBANS) was the primary outcome instrument for this study. ${ }^{20}$ The 12 -item RBANS consists of five subsets, corresponding to five domains of neuropsychological process: 1) immediate memory (list learning and story memory); 2) visuospatial/constructional ability (figure copy and line orientation); 3) language (picture naming and semantic fluency); 4) attention (digit span and coding); and 5) delayed memory (list learning free recall, list learning recognition, story memory free recall, and figure free recall).

Cognitive function was evaluated using the RBANS at pretest (baseline) and post-test, and at the 6-month, 12-month and, finally, 18-month follow-ups (endpoint). The RBANS tests were evaluated by two trained physicians. The assessors who carried out the clinical evaluation of the subjects were blinded to their allocation status. The consistency coefficiency Kappa was 0.87, guaranteeing inter-rater consistency. Data were reviewed after being entered into the database.

The severity of psychotic symptoms exhibited by the schizophrenia patients was evaluated using the PANSS at baseline and endpoint in order to evaluate the clinical status of schizophrenia. PANSS evaluations were conducted by two experienced psychiatrists who had simultaneously attended a training session in the use of PANSS. Repeated assessments found that a correlation coefficient of more than 0.8 was maintained for the PANSS total scores. ${ }^{21}$

\section{Statistical analysis}

All statistical analyses were performed using SPSS 17.0 (IBM Corporation, Armonk, NY, USA). The patient's demographic and clinical variables were compared between groups by use of independent $t$-tests for quantitative variables and Fisher's exact test for qualitative variables. Analysis of covariance was used to compare the RBANS and PANSS results between groups, controlling for demographic and clinical characteristics. Effect sizes were used to quantify the magnitude of the difference in RBANS and PANSS scores between the CFT and UDC groups, as well as to quantify the change in RBANS scores during the follow-up period between the groups. All $P$-values are two-tailed, with $P<0.05$ being considered statistically significant.

\section{Results Participant flow}

Figure 1 illustrates the trial profile. In total, 256 eligible patients were randomized to the treatment groups, with 133 allocated to the CFT and 123 to the UDC. By the endpoint, seven patients had dropped out from the former group and 15 from the latter, leaving a final experimental sample of 126 patients in the CFT and 108 patients in the UDC.

\section{Demographic and clinical characteristics}

Table 1 shows the demographic and clinical characteristics of the patient groups at the baseline assessment. There were no significant differences between the CFT and UDC groups for all demographic characteristics including sex, age, education, age at onset, and duration of illness.

\section{Comprehensive therapy outcome}

At the baseline assessment, the CFT and UDC groups had respective average RBANS total scores of $183.87 \pm 37.32$ and $178.55 \pm 32.95$, with no significant difference in cognitive function between the two groups (Table 2). During the 18-month follow-up observation, the change in RBANS total score indicated that patients in the CFT group showed greater improvement from baseline to the follow-up assessments in terms of cognitive function when compared to those in the UDC group ( $P=0.002$; Table 3$)$. Post hoc analysis further showed that those in the CFT group presented with significant differences in the RBANS total score, immediate memory, visuospatial skill, language, attention, and delayed memory sections as compared with those in the UDC group after 18 months of follow-up (all $P<0.01$; Table 2). After comparing the difference in PANSS scores between the CFT and UDC groups, we found no significant difference at either the baseline or endpoint visits (Table 4).

\section{Discussion}

To explore an effective paradigm of cognitive rehabilitation for schizophrenia, we evaluated the effect of CFT on cognitive function in the patients. To the best of our knowledge, this is the first study to investigate the impact of CFT on ameliorating cognitive dysfunction in stabilized patients with schizophrenia in the community. Our results showed that the dropout rate ( $8.6 \%$ at endpoint) of the study is considerably low, implying that CFT may be an acceptable paradigm to 


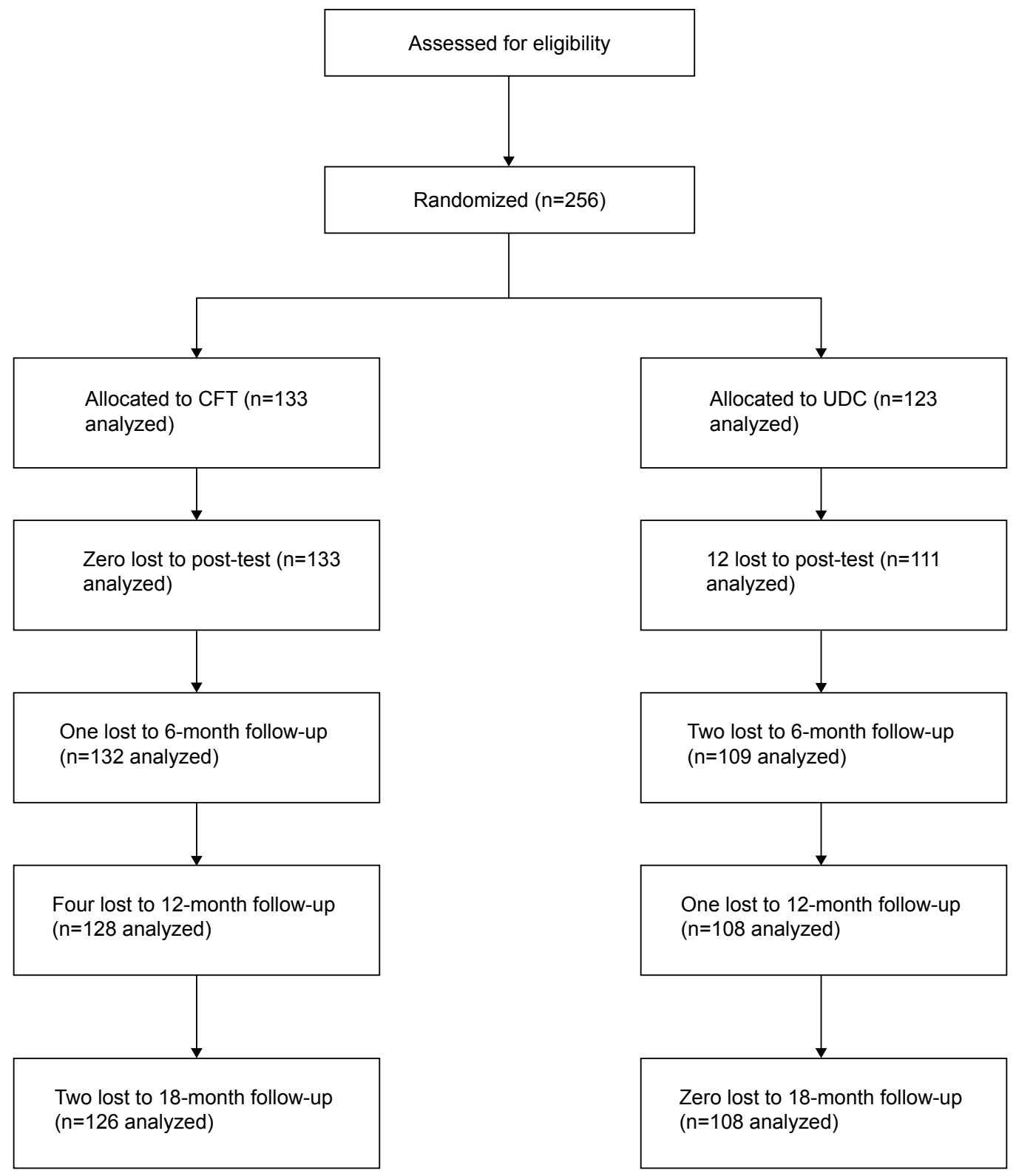

Figure I Flow diagram of the progress through the phases of the randomized trial.

Abbreviations: n, number; CFT, comprehensive family therapy group; UDC, usual daily care group.

Table I Demographic and clinical characteristics between the CFT and UDC groups at baseline

\begin{tabular}{|c|c|c|c|c|}
\hline Variable & CFT $(n=133)$ & UDC $(n=123)$ & Statistics & $P$-value \\
\hline \multicolumn{5}{|l|}{ Sex } \\
\hline Male & 68 & 51 & $\chi^{2}=2.40$ & 0.12 \\
\hline Female & 65 & 72 & & \\
\hline Age (years) ${ }^{\mathrm{a}}$ & $33.92 \pm 9.03$ & $34.49 \pm 8.92$ & $t=-0.5 \mathrm{I}$ & 0.51 \\
\hline \multicolumn{5}{|l|}{ Education } \\
\hline Middle school & 42 & 48 & $\chi^{2}=2.42$ & 0.30 \\
\hline High school & 69 & 52 & & \\
\hline College & 22 & 23 & & \\
\hline Age at onset (years) ${ }^{\mathrm{a}}$ & $29.94 \pm 8.68$ & $30.76 \pm 8.82$ & $t=-0.75$ & 0.93 \\
\hline Duration of illness (months) ${ }^{\mathrm{a}}$ & $47.38 \pm|6.5|$ & $44.32 \pm 16.39$ & $t=1.49$ & 0.45 \\
\hline
\end{tabular}

Note: ${ }^{\mathrm{D} D a t a}$ are presented as the mean \pm standard deviation.

Abbreviations: CFT, comprehensive family therapy group; UDC, usual daily care group; n, number. 
Table 2 RBANS score at baseline and endpoint visits

\begin{tabular}{|c|c|c|c|c|c|c|c|c|}
\hline RBANS & Baseline & $F^{a}$ & $P^{a}$ & $\mathbf{E S}^{\mathbf{a}}$ & Endpoint & $F^{b}$ & $P^{b}$ & $\mathbf{E S}^{\mathrm{b}}$ \\
\hline \multicolumn{9}{|c|}{ Total score } \\
\hline CFT & $\mid 83.87 \pm 37.32$ & 1.42 & 0.24 & 0.01 & $211.83 \pm 35.84$ & 22.92 & $<0.001$ & 0.09 \\
\hline UDC & $178.55 \pm 32.95$ & & & & $189.23 \pm 36.19$ & & & \\
\hline \multicolumn{9}{|c|}{ Immediate memory } \\
\hline CFT & $33.32 \pm 10.53$ & 1.04 & 0.31 & 0.04 & $40.02 \pm 10.42$ & 18.35 & $<0.001$ & 0.07 \\
\hline UDC & $32.03 \pm 9.26$ & & & & $34.69 \pm 8.54$ & & & \\
\hline \multicolumn{9}{|c|}{ Visuospatial skill } \\
\hline CFT & $29.32 \pm 6.65$ & 1.53 & 0.22 & 0.01 & $32.63 \pm 5.35$ & 13.57 & $<0.001$ & 0.06 \\
\hline UDC & $28.49 \pm 6.95$ & & & & $29.83 \pm 6.00$ & & & \\
\hline \multicolumn{9}{|l|}{ Language } \\
\hline CFT & $25.79 \pm 5.25$ & 0.19 & 0.89 & 0.00 & $29.44 \pm 5.94$ & 9.35 & 0.003 & 0.04 \\
\hline UDC & $25.73 \pm 4.46$ & & & & $27.02 \pm 5.80$ & & & \\
\hline \multicolumn{9}{|l|}{ Attention } \\
\hline CFT & $55.76 \pm 18.62$ & 0.28 & 0.60 & 0.00 & $63.33 \pm 15.99$ & 8.14 & 0.005 & 0.04 \\
\hline UDC & $54.25 \pm 15.84$ & & & & $56.92 \pm 15.96$ & & & \\
\hline \multicolumn{9}{|c|}{ Delayed memory } \\
\hline CFT & $39.68 \pm 9.96$ & 2.40 & 0.12 & 0.01 & $46.41 \pm 10.22$ & 19.77 & $<0.001$ & 0.07 \\
\hline UDC & $38.05 \pm 9.92$ & & & & $40.78 \pm 10.30$ & & & \\
\hline
\end{tabular}

Notes: Data are presented as the mean \pm standard deviation. ${ }^{a} A t$ baseline; bat endpoint.

Abbreviations: RBANS, Repeatable Battery for the Assessment of Neuropsychological Status; ES, effect size; CFT, comprehensive family therapy group; UDC, usual daily care group.

improve cognitive function among schizophrenia patients. We also found that CFT resulted in a significant improvement in cognitive function, and that this improvement was maintained at least over an interval of 18 months.

Earlier studies documented that individuals with schizophrenia exhibited significant impairments in cognitive functions, and that these impairments are strongly related to social outcomes. ${ }^{22}$ SSIT was previously found to be an effective strategy to improve social impairments in schizophrenia; ${ }^{19}$ in clinical practice, SSIT was found to improve cognitive function in patients with schizophrenia, ${ }^{23}$ which suggests that cognitive rehabilitation may be an important factor for social improvement. However, a recent observation indicated that no significant persistent change in cognitive function occurred when only SSIT was carried out. ${ }^{23}$ Here, we integrated SSIT and

Table 3 RBANS total scores at each visit and the results of repeated measures analysis of variance (visit $\times$ group) for the CFT and UDC groups

\begin{tabular}{llllll}
\hline Visit & CFT & UDC & F & P & ES \\
\hline Pretest & $183.87 \pm 37.32$ & $178.55 \pm 32.95$ & 9.77 & 0.002 & 0.04 \\
Pro-test & $195.68 \pm 36.17$ & $184.45 \pm 33.58$ & & & \\
6 months & $203.20 \pm 38.02$ & $189.09 \pm 34.95$ & & & \\
12 months & $206.91 \pm 34.40$ & $188.31 \pm 33.76$ & & & \\
18 months & $211.83 \pm 35.84$ & $189.23 \pm 36.19$ & & & \\
\hline
\end{tabular}

Note: Data presented as the mean \pm standard deviation.

Abbreviations: RBANS, Repeatable Battery for the Assessment of Neuropsychological Status; CFT, comprehensive family therapy group; UDC, usual daily care group; ES, effect size. family intervention as a whole therapy paradigm for patients with schizophrenia.

Our rationale for adding family intervention to the more typical SSIT treatment is based on several lines of evidence. First, family intervention has been considered an important part of modern treatment methods besides drugs in schizophrenia treatment and care. ${ }^{24}$ Second, family intervention promotes the development of coping abilities among both patients and their family members by providing information about the treatment and care of schizophrenia. ${ }^{25}$ Third, family intervention has been proven to be effective in treating people with schizophrenia within the People's Republic of China, ${ }^{26}$ where family members are more involved patients' care, which is also the case in many other developing countries. ${ }^{27}$ Our present findings suggest that this integrated intervention approach reached the goal of achieving persistent improvements in cognitive function among patients with schizophrenia in the community. While we cannot indicate whether this therapy paradigm can be applied in other countries, the present results provide evidence that this paradigm is practical in certain settings. More importantly, our results suggest that this combination therapy results in better efficacy in improving cognitive functions for schizophrenia patients in the People's Republic of China, as compared with only taking to medications alone.

We further evaluated the effects of CFT on the symptomatology in schizophrenia patients, and found that the psychopathology of both groups did not improve significantly 
Table 4 PANSS score at baseline and endpoint visits

\begin{tabular}{|c|c|c|c|c|c|c|c|c|}
\hline PANSS & Baseline & $F^{a}$ & $P^{a}$ & $\mathbf{E S}^{\mathbf{a}}$ & Endpoint & $F^{\mathrm{b}}$ & $P^{b}$ & $\mathbf{E S}^{\mathrm{b}}$ \\
\hline \multicolumn{9}{|l|}{ Total } \\
\hline CFT & $46.35 \pm 9.21$ & 1.75 & 0.19 & 0.01 & $41.91 \pm 9.49$ & 3.00 & 0.08 & 0.01 \\
\hline UDC & $44.96 \pm 8.65$ & & & & $40.10 \pm 7.48$ & & & \\
\hline \multicolumn{9}{|c|}{ Positive symptom } \\
\hline CFT & $10.53 \pm 3.24$ & 1.24 & 0.27 & 0.03 & $9.44 \pm 2.87$ & 1.59 & 0.21 & 0.01 \\
\hline UDC & $10.11 \pm 3.24$ & & & & $9.03 \pm 2.45$ & & & \\
\hline \multicolumn{9}{|c|}{ Negative symptom } \\
\hline CFT & $12.59 \pm 3.84$ & 0.87 & 0.35 & 0.03 & $\mid I .1 \mathrm{I} \pm 3.83$ & 1.10 & 0.30 & 0.01 \\
\hline UDC & $12.14 \pm 3.98$ & & & & $10.62 \pm 3.45$ & & & \\
\hline \multicolumn{9}{|c|}{ General psychopathology } \\
\hline CFT & $23.23 \pm 4.67$ & 1.00 & 0.32 & 0.01 & $21.36 \pm 4.34$ & 3.71 & 0.06 & 0.01 \\
\hline UDC & $22.7 I \pm 4.46$ & & & & $20.46 \pm 3.58$ & & & \\
\hline
\end{tabular}

Notes: Data are presented as the mean \pm standard deviation. ${ }^{a} \mathrm{At}$ baseline; bat endpoint.

Abbreviations: PANSS, Positive and Negative Syndrome Scale; ES, effect size; CFT, comprehensive family therapy group; UDC, usual daily care group.

in the interval between pretreatment and the 18-month follow-up. This result may suggest that CFT does not have a therapeutic effect on symptomatology. However, it should be noted that the participants included in this study were stable schizophrenia patients with a PANSS total score of less than 60. As such, we cannot fully exclude the influence of CFT on psychopathology. Further investigations are needed to evaluate the effects of CFT on clinical consequences in patients with an acute episode of schizophrenia.

\section{Limitations}

There are several limitations that should be noted. First, this is an 18-month follow-up trial. In future, a longer-term clinical trial would substantially contribute to our understanding of the longer-term effects of comprehensive intervention on cognitive outcomes. Second, the absence of an active control group (eg, a treatment program without a family intervention) may represent a limitation. Therefore, a randomized controlled trial with an active control group needs to be carried out to validate our findings. Also, the evaluation of family outcomes (eg, experienced burden) is helpful to explore this issue in more depth. Third, a learning effect with RBANS cannot be fully excluded, but such an effect is likely to be modest after 6 months, and it may affect both groups equally. ${ }^{28}$ Fourth, we did not record the use of antipsychotic medication during the follow-up period. This is likely to influence the results of RBANS because previous studies showed that cognitive function in patients with schizophrenia may be affected by antipsychotics. ${ }^{8,29,30}$ Finally, a limitation of this study was the open-label design, which precluded us from making direct comparisons between the two groups after 18 months. Nevertheless, it provided clinical data supporting the effectiveness of CFT on cognitive rehabilitation in schizophrenia.

\section{Conclusion}

According to our present findings, CFT is easily adaptable and may provide a promising approach to improving cognitive function in patients with schizophrenia. These results also support the idea that the combined program provides an effective paradigm for cognitive rehabilitation among individuals with schizophrenia in the community.

\section{Acknowledgments}

We are deeply grateful to all participants. We thank two anonymous reviewers for their insightful comments. This work was supported by the National Natural Science Foundation of China (81471358), the Shanghai Science and Technology Commission (14411969000), the Shanghai Health System Advanced and Appropriate Technology Promotion Project (2013SY012), and the National Key Clinical Disciplines at Shanghai Mental Health Center (OMA-MH, 2011-873).

\section{Disclosure}

The authors report no conflicts of interest in this work.

\section{References}

1. Zhang C, Li Z, Shao Y, et al. Association study of tryptophan hydroxylase-2 gene in schizophrenia and its clinical features in Chinese Han population. J Mol Neurosci. 2011;43(3):406-411.

2. Long J, Huang G, Liang W, et al. The prevalence of schizophrenia in mainland China: evidence from epidemiological surveys. Acta Psychiatr Scand. 2014;130(4):244-256.

3. Paquin K, Wilson AL, Cellard C, Lecomte T, Potvin S. A systematic review on improving cognition in schizophrenia: which is the more commonly used type of training, practice or strategy learning? BMC Psychiatry. 2014;14:139.

4. Keefe RS, Harvey PD. Cognitive impairment in schizophrenia. Handb Exp Pharmacol. 2012;(213):11-37.

5. Lu W, Zhang C, Yi Z, Li Z, Wu Z, Fang Y. Association between BDNF Val66Met polymorphism and cognitive performance in antipsychotic-naïve patients with schizophrenia. J Mol Neurosci. 2012; 47(3):505-510. 
6. Zhang C, Cai J, Zhang J, et al. Genetic modulation of working memory deficits by ankyrin 3 gene in schizophrenia. Prog Neuropsychopharmacol Biol Psychiatry. 2014;50:110-115.

7. Elvevåg B, Goldberg TE. Cognitive impairment in schizophrenia is the core of the disorder. Crit Rev Neurobiol. 2000;14(1):1-21.

8. Zhang C, Fang Y, Xu L. Glutamate receptor 1 phosphorylation at serine 845 contributes to the therapeutic effect of olanzapine on schizophrenialike cognitive impairments. Schizophr Res. 2014;159(2-3):376-384.

9. Marder SR, Fenton W. Measurement and Treatment Research to Improve Cognition in Schizophrenia: NIMH MATRICS initiative to support the development of agents for improving cognition in schizophrenia. Schizophr Res. 2004;72(1):5-9.

10. Shimizu S, Mizuguchi Y, Ohno Y. Improving the treatment of schizophrenia: role of 5-HT receptors in modulating cognitive and extrapyramidal motor functions. CNS Neurol Disord Drug Targets. 2013;12(6):861-869.

11. Cai J, Yi Z, Lu W, Fang Y, Zhang C. Crosstalk between 5-HT2cR and PTEN signaling pathway in atypical antipsychotic-induced metabolic syndrome and cognitive dysfunction. Med Hypotheses. 2013;80(4) 486-489.

12. Genevsky A, Garrett CT, Alexander PP, Vinogradov S. Cognitive training in schizophrenia: a neuroscience-based approach. Dialogues Clin Neurosci. 2010;12(3):416-421.

13. Dang J, Zhang J, Guo Z, et al. A pilot study of iPad-assisted cognitive training for schizophrenia. Arch Psychiatr Nurs. 2014;28(3):197-199.

14. Roberts DL, Penn DL. Social cognition and interaction training (SCIT) for outpatients with schizophrenia: a preliminary study. Psychiatry Res. 2009;166(2-3):141-147.

15. Bowie CR, McGurk SR, Mausbach B, Patterson TL, Harvey PD. Combined cognitive remediation and functional skills training for schizophrenia: effects on cognition, functional competence, and realworld behavior. Am J Psychiatry. 2012;169(7):710-718.

16. Friedman-Yakoobian MS, Mueser KT, Giuliano A, GoffDC, Seidman LJ. Family-directed cognitive adaptation for schizophrenia. J Clin Psychol. 2009;65(8):854-867.

17. American Psychiatric Association. Diagnostic and statistical manual of mental disorders: DSM-IV. 4th ed. Washington, DC: American Psychiatric Association; 1994.

18. Cheng Y, Wu W, Feng W, et al. The effects of multi-domain versus single-domain cognitive training in non-demented older people: a randomized controlled trial. BMC Med. 2012;10:30.
19. Liberman RP, Wallace CJ, Blackwell G, Kopelowicz A, Vaccaro JV, Mintz J. Skills training versus psychosocial occupational therapy for persons with persistent schizophrenia. Am J Psychiatry. 1998;155(8): 1087-1091.

20. Randolph C, Tierney MC, Mohr E, Chase TN. The Repeatable Battery for the Assessment of Neuropsychological Status (RBANS): preliminary clinical validity. J Clin Exp Neuropsychol. 1998;20(3):310-319.

21. Zhu Y, Wang Z, Ni J, et al. Genetic variant in NDUFS1 gene is associated with schizophrenia and negative symptoms in Han Chinese. J Hum Genet. 2015;60(1):11-16.

22. Pinkham AE. Social cognition in schizophrenia. J Clin Psychiatry. 2014;75 Suppl 2:14-19.

23. Bucci P, Piegari G, Mucci A, et al. Neurocognitive individualized training versus social skills individualized training: a randomized trial in patients with schizophrenia. Schizophr Res. 2013;150(1):69-75.

24. Cohen AN, Glynn SM, Murray-Swank AB, et al. The family forum: directions for the implementation of family psychoeducation for severe mental illness. Psychiatr Serv. 2008;59(1):40-48.

25. Ozkan B, Erdem E, Demirel Ozsoy S, Zararsiz G. Effect of psychoeducation and telepsychiatric follow up given to the caregiver of the schizophrenic patient on family burden, depression and expression of emotion. Pak J Med Sci. 2013;29(5):1122-1127.

26. Chien WT, Chan SW. The effectiveness of mutual support group intervention for Chinese families of people with schizophrenia: a randomised controlled trial with 24-month follow-up. Int J Nurs Stud. 2013;50(10):1326-1340.

27. Guo X, Zhai J, Liu Z, et al. Effect of antipsychotic medication alone vs combined with psychosocial intervention on outcomes of early-stage schizophrenia: A randomized, 1-year study. Arch Gen Psychiatry. 2010; 67(9):895-904.

28. Pontes LM, Martins CB, Napolitano IC, et al. Cognitive training for schizophrenia in developing countries: a pilot trial in Brazil. Schizophr Res Treatment. 2013;2013:321725.

29. Han M, Zhang XY, Chen da C, et al. Cognitive differences in schizophrenia on long-term treatments with clozapine, risperidone and typical antipsychotics. Int Clin Psychopharmacol. 2015;30(2):89-95.

30. Beninger RJ, Baker TW, Florczynski MM, Banasikowski TJ. Regional differences in the action of antipsychotic drugs: implications for cognitive effects in schizophrenic patients. Neurotox Res. 2010;18(3-4) $229-243$.
Neuropsychiatric Disease and Treatment

\section{Publish your work in this journal}

Neuropsychiatric Disease and Treatment is an international, peerreviewed journal of clinical therapeutics and pharmacology focusing on concise rapid reporting of clinical or pre-clinical studies on a range of neuropsychiatric and neurological disorders. This journa is indexed on PubMed Central, the 'PsycINFO' database and CAS,

\section{Dovepress}

and is the official journal of The International Neuropsychiatric Association (INA). The manuscript management system is completely online and includes a very quick and fair peer-review system, which is all easy to use. Visit http://www.dovepress.com/testimonials.php to read real quotes from published authors. 